\title{
Educación Física de Calidad desde la perspectiva de la práctica docente: propiedades psicométricas de un instrumento para su evaluación Quality Physical Education from the perspective of teaching practice: psychometric properties of an instrument for its evaluation
}

* Javier Rodríguez Rodríguez, *0 swaldo Ceballos Gurrola, *Jorge Isabel Zamarripa Rivera, *Rosa Elena M edina Rodríguez, **Walter Ho, ***Rosa López D 'Amico

*U niversidad Autónoma de Nuevo León (M éxico), **Universidad de Macao (Macao), ***U niversidad Pedagógica Experimental Libertador (Venezuela)

Resumen. El propósito del estudio fue examinar las propiedades psicométricas y estructura factorial del Cuestionario de Educación Física de Calidad traducido y adaptado al español y contexto mexicano. Se presenta un estudio cuantitativo con un diseño instrumental. Participaron 763 docentes de Educación Física de M éxico con rango de edad 19 a 71 años (M = 37.89 \pm 11.18 ); donde el $31.7 \%$ son mujeres y $68.3 \%$ hombres. El $59.4 \%$ trabajan en escuelas que pertenecen al sistema federal, el 33.9\% al sistema estatal y el $6.7 \%$ trabajan en escuelas particulares. Se realizó la traducción y adaptación al español y contexto mexicano de la encuesta de Educación Física de Calidad compuesta por 50 ítems que se responden sobre una escala que va desde 0 «no al canzado totalmente» y 10 «completamenteal canzado». Se efectuó el análisisfactorial exploratorio (AFE) utilizando la matriz de correlaciones Kaiser-M eyer O Ikin KM O (.97) y prueba de esfericidad de Bartlett ( $x^{2} \times 2=30645.936$ y $\mathrm{gl}=990$ ) obteniendo seis factores y la reducción a 46 ítems. El análisis factorial confirmatorio (AFC) mostró una buena consistencia interna y correlaciones de inter factor $\left(\mathrm{x}^{2} / \mathrm{gl}=2.98, \mathrm{NNFI}=.98, \mathrm{CFI}=.98\right.$ y RMSEA $\left.=.07\right)$, aś como al dividirlo por género, nivel y sistema educativo. El instrumento muestra buenas propiedades psicométricas para valorar la Educación Física de Calidad desde la per spectiva de la práctica docente en el contexto mexicano. Por sus características podría utilizarse en otros países de habla hispana ya que su redacción, terminología y contenidos se adaptan al contexto.

Palabras clave: Calidad en la Educación, Políticas Educativas, Educación Física, Práctica docente, Validación de escala.

Abstract. The purpose of the study was to examine the psychometric properties and factor structure of the quality Physical Education scale from the perspective of teaching practice, translated and adapted to the Spanish and Mexican context. A quantitative study with an instrumental design is presented. The participants were 763 Physical Education teachers from M exico with an age range of 19 to 71 years old ( $M=37.89 \pm 11.18$ ); where $31.7 \%$ were women and $68.3 \%$ men. $59.4 \%$ work in schools that belong to the federal system, $33.9 \%$ to the state system and $6.7 \%$ work in private schools. The translation and adaptation to Spanish and the M exican context of the Q uality Physical Education survey was carried out. It is composed of 50 items that are answered on a scal ethat goes from 0 «not totally achieved» to 10 «completely achieved». It was carried out the exploratory factorial analysis(EFA) using the Kaiser-M eyer O Ikin KMO correlation matrix (.97) and Bartlett's sphericity test $\left(\mathrm{x}^{22} \times 2=30645.936 \mathrm{y} \mathrm{gl}=990\right)$ obtaining six factors and the reduction to 46 items. Confirmatory factor analysis (CFA) showed good internal consistency and inter-factor correlations $\left(x^{2} / g l=2.98, \mathrm{~N} \mathrm{NFI}=.98, \mathrm{CFI}=.98\right.$ and RMSEA $\left.=.07\right)$, as well as when divided by gender, level and educational system. The instrument shows good psychometric properties to evaluate $Q$ uality Physical Education from the perspective of teaching practice in the M exican context. Due to its characteristics, it could be used in other Spanish-speaking countries since its writing, terminology and contents are adapted to the context. Keywords: Educational policies, Physical Education, Q uality in Education, Teaching practice, Validation of scale.

\section{Introducción}

LaEducación Física a lo largo de su historia habusca do la mejora continua para elevar su calidad. De manera particular directivos escolares y docentes en su práctica diaria implementan estrategias para brindar una

Fecha recepción: 24-11-20. Fecha de aceptación: 05-01-21

O swaldo Ceballos Gurrola

oscegu@ hotmail.com educación integral en el alumno. Por otro lado, y con un alcance más global, la O rganización de las Naciones U nidas para la Educación, la Ciencia y la Cultura (UNESCO) convoca organizaciones internacionales comoThe International Society for Comparative PE and Sport (ISCPES), International Association of Physical Education and Sport for Girls andW omen (IAPESGW ), International Federation of Adapted Physical Activity (IFAPA) and Federation Internationale d' Education Physique (FIEP), para que en reunión de ministros del 
deporte puedan discutir el desarrollo de la Educación Física. Producto de este trabajo, se elaboraron políticas de Educación Física de cal idad inclusiva con documentos que han sido la guía y metodología para su implementación especialmente en países en desarrollo (UNESCO, 2015).

La Educación Física de calidad es la experiencia de aprendizaje planificada, progresiva e inclusiva que forma parte del currículo en la educación básica. En este sentido, actúa como punto de partida para desarrollar hábitos de actividad física y deporte en cada una de sus etapas de vida. La experiencia de aprendizaje que se ofrece a los niños y jóvenes a través de las clases de Educación Física debe ser apropiada para ayudarles a adquirir las habilidades psicomotrices, la comprensión cognitiva y las aptitudes sociales y emocionales que necesitan para llevar una vida físicamente activa (UNESCO, 2015).

La finalidad de la Educación Física (como área curricular específica dentro de un sistema educativo universal, obligatorio y público), es el desarrollo físicomotriz del alumnado, la promoción de la culturafísicay su aportación al planteamiento global de desarrollo integral, como ciudadanos de una sociedad democrática, fundamentalmente en el desarrollo de las competencias clave y en el desarrollo integral del alumnado desde, con y a través de la motricidad (López, Pérez, M anrique\& M onjas, 2016). LaEducación Física, por tanto, debería llegar a todos los jóvenes en igualdad de condiciones independientemente del género, raza, estatus socioeconómico, entre otros (Gil-Espinosa $\&$ AznarCebamanos, 2016).

Por otro lado, la inactividad física constituye una pandemia global, de modo que diversas organizaciones internacional es han hecho un Ilamado a la acción con el fin de tratar esta problemática (Guthold et al., 2018). En México, los datos registrados señalan que más del $80 \%$ de los niños de 10 a 14 años y cerca del $40 \%$ de los adolescentes de 15 a 19 años son inactivos, las evidencias indican que este factor de riesgo está en aumento. Durante el 2013, la inactividad física en M éxico tuvo un costo aproximado de 16 mil millones de pesos. En este sentido, la Educación Física de calidad es una estrategia de probada eficacia, puede fomentar el compromiso con la actividad física e incrementar sus niveles entre la población a lo largo de la vida (Jauregui, 2018).

La Boleta de Calificaciones M exicana sobre laActividad Física de Niños y Jóvenes Galaviz et al., (2018) muestra datos que son preocupantes, ya que en M éxico hay 207.682 escuelas públicas de educación básica para
96.000 profesores de Educación Física, donde solo el $36.3 \%$ de los escolares recibe una hora de Educación Física a la semana. Por otro lado, existen pocas 0 rganizaciones no Gubernamentales (ONG) que apoyan la promoción de actividad física. Las iniciativas implementadas por ONG están dirigidas a promover el deporte competitivo, los programas son de corta duración y se desconoce la proporción de niños y jóvenes que estos al canzan.

Las políticas para una educación física de calidad han sido motivo de análisispor parte delaUNESCO (2015), fundamental para el desarrollo integral de las personas, la adquisición de las competencias clave y asimilación de un estilo de vida saludable (Gil-Espinosa $\&$ AznarCebamanos, 2016), en ella se ve la necesidad de contar con un curriculum desde un enfoque integral, inclusivo, flexible, adaptablealos cambios educativosy con lamayor cobertura posible. Algunos autores manifiestan, además, la necesidad de atender los retos de la Educación física sobre lo que debe de aprender el alumno, la transferencia del aprendizaje escolar a la vida durante y después de la escuela (López et al., 2016). U no de los autores que ha profundizado en el tema de estudio es Walter $\mathrm{Ho}$, ya sea para poner a prueba y validar escalas sobre la percepción del docente para una Educación Física de calidad en Asia (Ho et al, 2019), Latinoamérica (Ho et al. , 2018) y Europa (Ho et al. , 2019); o compartiendo las perspectivas globales de la Educación Física y el deporte (Ho et al., 2016).

La información antes expuesta da muestra de los beneficios de la Educación Física, desarrollo de políticas, igualdad de condiciones; así como los problemas asociados a la inactividad física y falta de cobertura para atender la educación básica. Por todo ello, se propone como objetivo de estudio examinar las propiedades psicométricas y estructura factorial del Cuestionario de Educación Física de Calidad traducido y adaptado al español y contexto mexicano.

\section{Método}

\section{Tipo de estudio y participantes}

El presente estudio tiene un enfoque cuantitativo de carácter descriptivo y correlacional. Participaron de manera aleatoria y voluntaria 763 docentes de Educa ción Física de la República M exicana con un rango de edad 19 y 71 años $(M=37.88 \pm 11.70)$; dondeel $31.7 \%$ son mujeres y $68.3 \%$ hombres. De manera específica, el $12.5 \%$ labora en educación preescolar, $48.4 \%$ en primaria y $39.1 \%$ en secundaria. Respecto al tipo de 
centro educativo, la mayoría pertenecen al sistema federal $(59.4 \%)$, seguido por los de tipo estatal (33.9\%) y privado $(6.7 \%)$.

\section{Instrumento}

Se utilizó encuesta sobre el Índice Global de la Educación Física de Calidad (GIQPE, por sus siglas en inglés) de $\mathrm{Ho}$ et al. (2018) utilizada en estudios previos con poblaciones de docentes latinoamericanos y asiáticos, obteniendo adecuados val ores de fiabilidad (á> .85) e eíndices de bondad y ajuste aceptables (Ho et al., 2019) - La encuesta está compuesta de 50 ítems en torno a la Educación Física de calidad, agrupados en ocho factores: desarrollo de habilidades y conciencia del cuerpo en las actividades físicas y deportivas; lugar, instalaciones y equipo de educación física; enseñanza de la educación física; planes para la factibilidad y accesibilidad de la educación física para todos los estudiantes; normas sociales y práctica cultural; aportación gubernamental para el mejoramiento de la calidad de la educación física; habilidad cognitiva, pensamiento independiente y otras habilidades de desarrollo genérico; desarrollo de programas alternativos en educación física.

El instrumento está dividido en dos partes, en la primera se detallan los datos sociodemográficos (ciudad, género, institución, años de experiencia laboral, nivel donde trabaja y tipo de escuela), y en la segunda, las preguntas sobre la percepción de la Educación Física de cal idad en su entorno/ ciudad, con una escala de respuesta donde 1 = no alcanzado totalmente y $10=$ completamente alcanzado. El proyecto fue aprobado y registrado por la Coordinación de Investigación de la Fa cultad de O rganización Deportiva de la U niversidad Autónoma de Nuevo León (REPRIN-FO D-43).

\section{Procedimiento}

El instrumento original en inglés fue traducido al español hablado en M éxico siguiendo el procedimiento backtranslation (Hambleton \& Kanjee, 1995) por un grupo de tres expertos, compuesto por especialistasque trabajan en el área de la Educación Física, uno de ellos con experiencia en la validación de instrumentos, un traductor especializado en el área de la actividad física y el deporte, y un profesor de Educación Física de una escuela de educación básica, para discutir las discrepancias de la traducción y revisar la primera versión del instrumento al español hablado en México. Esta versión del cuestionario se radujo nuevamente al inglés por uno de los profesionales, para contrastar ambas versiones del instrumento: la original en inglés y la traducción inversa. Se analizaron nuevamente las diferencias en las versiones para introducir los cambios necesarios en la versión traducida al español hablado en México, para facilitar la comprensión de los ítems logrando la versión final de cada una de las escalas.

U na vez llevado a cabo el backtranslation, se procedió a la utilización del Método Delphi como instrumento de validación de cuestionarios por grupos de expertos, el cual ha sido ampliamente utilizado en numerosos estudios y ámbitos de conocimiento (Reguant-Álvarez \& Torrado-Fonseca, 2016), inclusive en las Ciencias de la Actividad Física y el Deporte; siguiendo las recomendaciones de otros autores (Calabuig et al., 2007; Soto, Collado, Torres \& Padial, 2021), donde los integrantes deben cumplir con requisitos tales como: buen conocimiento del Método Delphi, ser investigadores académicos con relación al tema a estudiar y tener gran facilidad deintercomunicación al trabajar conjuntamente en otros estudios.

El grupo de expertos se ha conformado a partir de especialistas de una escuela formadora de docentes en Educación Física, integrado por dos doctoresy un máster en actividad física y deporte con orientación en Educa ción Física. El primer paso fue fijar como criterio fundamental de selección la competencia de los candidatos en el área del conocimiento en que se inserta la investigación sobre la base de su currículo personal. Todos revisaron la redacción de cada uno de los ítems haciendo las recomendaciones para una mejor comprensión, así como su relación con los factores del mismo instrumento.

La encuesta se capturó y administró a través de Google Forms, donde se solicitó en permiso a la Dirección de Educación Física y Deporte de la Secretaría de Educación para el envío de la encuesta a través de la base de datos de los docentes de Educación Física. U na vez aceptado el consentimiento informado, se pide que sea cuidadoso en la lectura y respuesta a la hora de indicar su opinión; finalmente, se agradece el tiempo dedicado.

\section{Análisis estadístico}

Los datos se procesaron mediante los programas estadísticos SPSS versión 24 y el programa LISREL 8.80 (Jöreskog \& Sorbom, 2006). Se realizó el análisisfactorial exploratorio (AFE) utilizando la matriz de correlaciones Kaiser-M eyer O Ikin KMO y prueba de esfericidad de Bartlett, mediante el método de extracción de componentes principales, utilizando un criterio de rotación ortogonal Varimax. La fiabilidad del instrumento se cal- 
culó a través del coeficiente Alfa de Cronbach. El análisis factorial confirmatorio (AFC), se realizó atendiendo la naturaleza ordinal de las variables, con el método de estimación de Componentes Principales (Bentler, 2005). Un coeficiente $\div 2$ / gl inferior a 3.0 representa un buen ajuste del modelo (Kline, 2005). Los índices de CFI y NNFI por encima de .90 indican un ajuste aceptable (Hu \& Bentler, 1999), para la RMSEA se consideraron valores satiffactorios menores a .08 (BatistaFoguet et al., 2004).

\section{Resultados}

En laTabla 1, se muestran los estadísticos descriptivos de los diferentes ítems que componen el cuestiona rio original, así como la asimetría y curtosis. El ítem 23 muestra el promedio más alto con $8.98 \pm 1.32$ y el más bajo corresponde al ítem 26 con $6.91 \pm 2.66$.

Para comprobar la adecuación del instrumento, se realizó un análisis factorial exploratorio sobre los 50 ítems; se comprobó la adecuación de la muestra, por medio de la prueba de esfericidad de Bartlett, y el índice de adecuación de la muestra de Kaiser-M eyer-O Ikin (KMO). El valor de la medida de adecuación muestral fue óptimo, con un índice de KM 0 de.967; y la prueba de Bartlett resultó estadísticamente significativa con un valor ( $\left.?^{2}=30645.936 \mathrm{y} \mathrm{gl}=990\right)$. Se extrajeron seis factores (F1 Políticas para el mejoramiento de la calidad educativa, F2 Enseñanza de la educación física, F3 Desarrollo de habilidades, F4 Propósitos y contenidos,

Tabla 1

Estadísticos descriptivos asimetría y curtosis de los ítems

Items

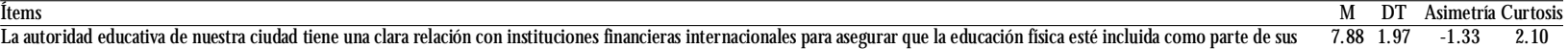

programas de ayuda en educación.

El programa de educación física de nuestra ciudad anima a los estudiantes a aprender e interactuar con los compañeros de clase.

Las escuel as en nuestra ciudad tienen instalaciones seguras y apropiadas para la enseñanza y el aprendizaje de la educación física

Nuestro programa de educación física contiene elementos que ayudan a desar rollar las destrezas básicas de los estudiantes para distintas actividades físicas y deportivas.

Las escuel as en nuestra ciudad tienen un ambiente seguro y apropiado para la enseñanza y el aprendizaje de la educación física.

Las actitudes positivas relacionadas con el deporte y los valores son el centro del aprendizaje en la educación física, en nuestra ciudad.

La autoridad educativa de la ciudad tiene una política clara de incentivar el desarrollo oportunidades de igualdad de aprendizaje en el programa de educación física de la escuela.

programa de educación física de nuestra ciudad incentiva a los estudiantes a asumir responsabilidades apropiadas para servir en clubes deportivos u otras actividades similares en la $8.04 \quad 1.89$

escuela 0 en la comunidad.

Nuestra ciudad tiene una política adecuada para reforzar la accesibilidad de la

El programa de educación física de nuestraciudad contiene elementos que ayudan a desarrollar en los estudiantes su comprensión adecuada de la salud y la aptitud física, incluyendo 8.54 1.61

El programa de educacion fica de nuestraciudad contiene elem

el establecimiento y logro de metas personal es para una vida sana.

El programa de educación física en las escuelas en nuestra ciudad ayuda a los estudiantes con el desarrollo de sus habilidades del pensamiento crítico. $\quad \begin{array}{llll}-1.31 & 2.55\end{array}$

Nuestra ciudad tiene una política apropiada extendiendo las oportunidades para el aprendizaje en actividades físicas a través de programas relacionados con actividades deportivas, $\quad 8.45 \quad \begin{array}{lll}1.88 & -1.84 & 3.97\end{array}$

programas de actividades después de clases, programas de actividades extracurriculares en las escuelas.

El programa de educación física de nuestra ciudad contiene oportunidades para acciones apropiadas para ayudar a los estudiantes a mantener estilos de vida activos y saludables. $\quad 8.92 \quad 1.72 \quad-2.39 \quad 6.90$

Nuestra autoridad educativa tiene un claro reconocimiento del rol característico de la educación física como parte de un sistema educativo balanceado para la realización del $\quad \begin{array}{llll}8.68 & 1.97 & -2.07 & 4.61\end{array}$

potencial humano, la salud y el bienestar de todos los ciudadanos.

El programa de educación física de nuestra ciudad ayuda a los estudiantes a desarrollar hábitos de ejercicio.

Los hábitos de educación física y deporte están bien desarrollados de acuerdo al programa de educación física de la escuela

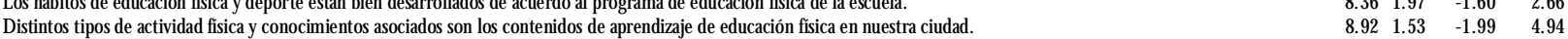

$\begin{array}{llll}8.65 & 1.67 & -1.86 & 4.38\end{array}$

$\begin{array}{llll}8.36 & 1.97 & -1.60 & 2.66\end{array}$

La autoridad en educación de la ciudad tiene planes de incentivar a cada estudiante a participar en deportes y actividades físicas luego de su horario escolar y a que use su tiempo libre $8.73 \quad 1.90 \quad-2.21 \quad 5.35$

sabiamente en deporte y actividades físicas.

Nuestra autoridad en educación tiene una política clara en cuanto al apoyo de investigaciones para mejorar la efectividad y cal idad de los programas de educación física en las escuelas $8.47 \quad 2.01$

El conocimiento en salud es considerado una de las áreas más importantes para aprender sobre la actividad física en nuestra ciudad.

El programa de educación física en nuestra ciudad ayuda al estudiante a desar rollarse competentemente en diferentes actividades físicas y deportivas.

Nuestro programa de educación física provee a estudiantes con oportunidades para que tengan parte en diferentes actividades físicas

Nuestro programa de educación física ayuda al estudiante a entender la relación entre actividades físicas y deportivas y desarrollo personal y social.

N uestro programa de educación física contiene elementos de enseñanza que permiten ayudar a estudiantes a comunicar afectivamente sus ideas y sentimientos con otros.

$\begin{array}{ll}\text { Nuestro programa de educación fisica contiene elementos de enseñanza que permiten ayudar a estudiantes a comunicar afectivamente sus ideas y sentimientos con otros. } & 8.73 \quad 1.44 \\ \text { Las escuel as en nuestra ciudad tienen un equipamiento acorde y seguro para la enseñanza y aprendizaje de la educación física } & 6.91 \\ 7.66\end{array}$

El conocimiento acerca del funcionamiento de nuestros cuerpos y su relación con las actividades están siendo bien enseñadas a través del programade educación física de la escuela. $7.37 \quad 2.39$

La autoridad de educación en nuestra ciudad reconoce que la educación física de buena calidad depende de educadores cualificados, por consiguiente, la prioridad es para el 8.352 .02

entrenamiento del personal calificados, aun cuando otras dependencias estén cortas en suministros.

La educación física es una materia obligatoria para los estudiantes de bachillerato en sus últimos años en nuestra ciudad.

El programa de educación física de las escuelas en nuestra ciudad cul tiva la habilidad del pensamiento independiente en el estudiante

La educación física es una materia obligatoria para los estudiantes de bachillerato en nuestra ciudad.

El conocimiento de actividades físicas y deporte están siendo bien enseñadas a través del programa de educación física de la escuela.

Los programas de educación física en las escuelas de nuestra ciudad realzan el desar rollo de la habilidad de los estudiantes para resolver problemas.

A los estudiantes en nuestra ciudad se les dan oportunidades adecuadas para aprender activamente durante las lecciones de actividad física.

La autoridad de educación de la ciudad tiene una política clara para lograr el desarrollo de que ambos géner os tengan oportunidades iguales para aprender en el programa de

educación física de la escuela

El conocimiento del crecimiento y su relación con actividades están bien impartidas a través del programa escolar de educación física.

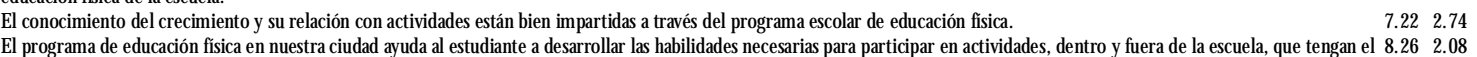

potencial para generar participación einvolucramiento a lo largo de la vida, disponibles en la comunidad.

El programa de educación física en las escuelas de nuestra ciudad real zala habilidad del pensamiento innovador del estudiante.

El programa de educación física en las escuelas de nuestra ciudad ayuda al estudiante a desarrollar el pensamiento moral y conduct

$\begin{array}{lllllll}\text { L a autoridad de educación en nuestra ciudad promueve planes de colaboración internacionales frecuentemente entre institutos para la preparación acerca de la calidad del desar rollo } & 8.11 & 2.63 & -1.46 & 2.33 & -1.61 & 2.85\end{array}$

de la educación física en las escuelas.

El programa de educación física en nuestra ciudad contiene elementos de enseñanza que ayudan a los estudiantes a desarrollar sus habilidades motoras básicas dentro del contexto de 8.601 .54

8.602 .01

$8.03 \quad 2.21$

$8.22 \quad 2.20$

7.271 .97

$7.822 .26-1.40-2.26$

$\begin{array}{llll}7.99 & 2.19 & -1.59 & 2.67\end{array}$ actividades físicas apropiadas.

El programa de educación física en nuestra ciudad es el medio más efectivo para equipar a los niños con habilidades, actitudes, valores, conocimiento, entendimiento y participación 8.801 .43

El programa de educación física en nuestra ciudad es el medio más efectivo para equipar a los niños con habilidades, actitudes, valores, conocimiento, entendimiento y participación $\quad 8.80 \quad 1.43 \quad-1.46 \quad 2.24$
en actividad física y deporte a lo largo de la vida.

programa de educación física de la escuela.

Todos los profesores de educación física en nuestra ciudad están calificados para enseñar educación física.

La educación física es una materia obligatoria para todos los estudiantes de primaria en nuestra ciudad.

La autoridad en educación de nuestra ciudad ha implementado políticas para la educación física como un elemento de derechos humanos para todos los niños.

$\begin{array}{llll}8.29 & 1.97 & -1.82 \quad 3.98\end{array}$

El desarrollo de las habilidades de los estudiantes está siendo efectivamente rasaca

$\begin{array}{llll}8.13 & 2.08 & -1.58 & 2.71 \\ 8.81 & 1.49 & -1.37 & 1.52\end{array}$

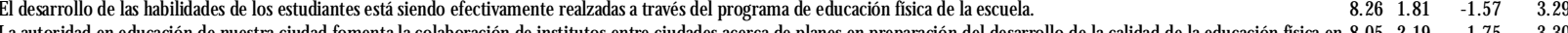

las escuelas.

El programa de educación física de nuestra ciudad contiene el ementos de enseñanza que ayuda al estudiante a desarrollar sus habilidades básicas en cuanto a la toma de decisiones y $\quad 8.51 \quad 1.61 \quad-1.46 \quad 2.83$

comunicación.

La enseñanza y el aprendizaje de la educación física en nuestras escuelas es divertida y agradable

\begin{tabular}{llll}
7.48 & 1.94 & -568 & -.594 \\
\hline
\end{tabular}

Nota: $M=$ Media DT = Desviación estándar 
F5 Planes para la factibilidad y accesibilidad para todos los estudiantes, F6 N ormas sociales y práctica cultura) y 45 ítems con valores Eigen de uno o mayores que, conjuntamente, explican un $70.68 \%$ de la varianza total acumulada (Tabla 2).

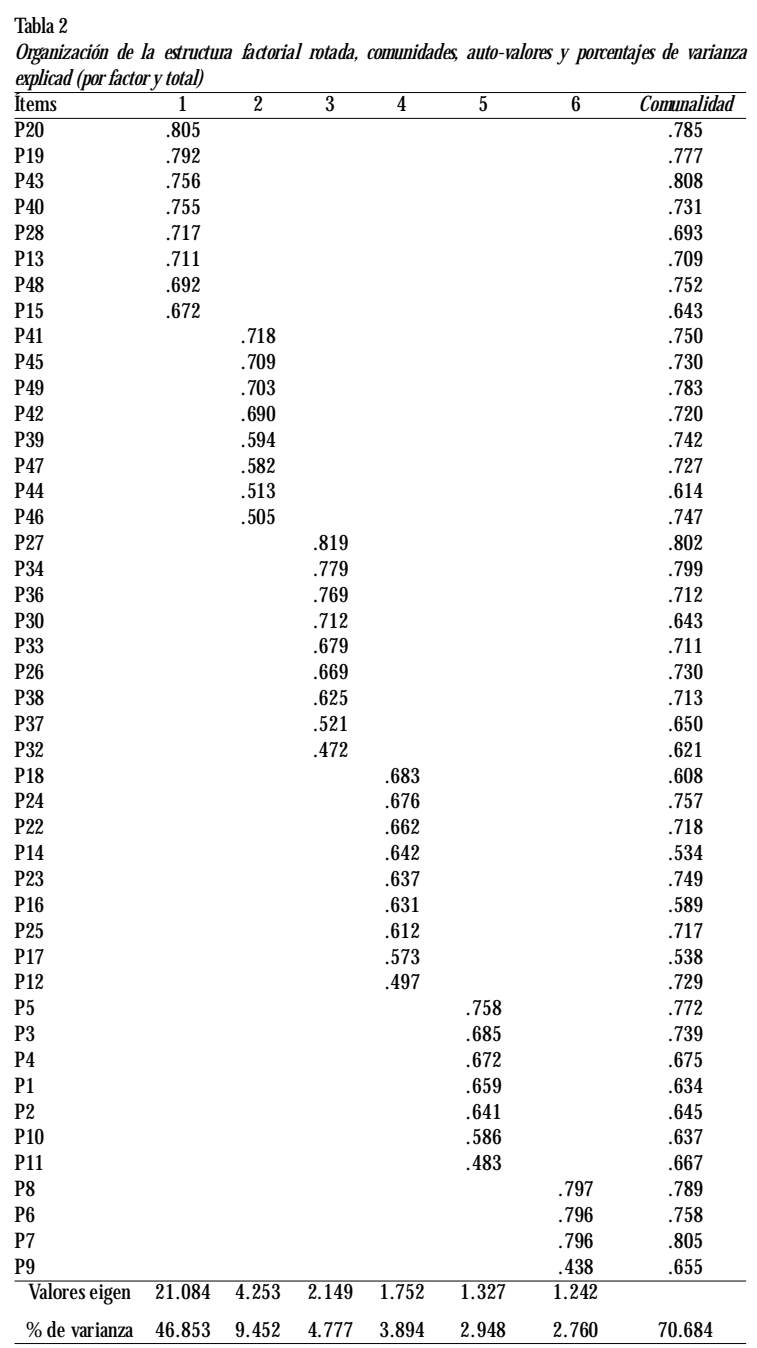

Una vez identificados los seis factores, se obtuvieron el valor promedio siendo el de enseñanza de la educación física el más al to (8.52 \pm 1.39$)$ y el más bajo el de normas sociales y práctica cultural (7.48 \pm 1.90$)$. La fia bilidad mediante el análisis de consistencia interna a tra vés del índice alfa de Cronbach, reveló una buena consistencia interna, mostrando valores en este caso superiores a 70 (Cronbach, 1951; 0 viedo y Campos-Arias, 2005) entre un rango de .85 y .94 (verTabla 3). El aná lisis de correlación mostró que todos los factores se

Tabla 3

Media, desviación estándar, fiabilidad, y correlaciones entre las variables

\begin{tabular}{llllllllll}
\hline Factores & Rango & $M$ & $D E$ & 1 & 2 & 3 & 4 & 5 & 6
\end{tabular}

\begin{tabular}{llllll}
\hline Políticas para el mejoramiento de & $1-10$ & 8.40 & 1.67 & $(.94)$
\end{tabular}

la calidad educativa

Enseñanza de la educación física $\quad 1-10 \quad 8.521 .39 .621 * *$ (.93)

Desar rollo de habilidades $\quad 1-10 \quad 7.76 \quad 1.84 .469 * * .765 * *$ (93)

Propósitos y contenidos $\quad 1-10 \quad 8.741 .21 .735 * * .749 * .634 * *(92)$

Planes para la factibilidad y $\quad \begin{array}{llllll}1 & & 8.17 & 1.44 & .644^{* *} .683^{* *} .611^{* *} .734 * * & (.90)\end{array}$

accesibilidad para los estudiantes

$\begin{array}{lllll}\text { Normas sociales y práctica cul tural } & 1-10 & 7.48 & 1.90 .376^{* *} .550 * * .571^{* *} .520^{* *} .607 * * & (.85)\end{array}$

Nota: M = M edia, DE= Desviación estándar, Alfa de Cronbach sobre la diagonal; $r$ de Pearson ** $p<.01$. correlacionan de manera positiva entre ellos y están significativamente relacionados $p<.01$.

Por lo anterior, los índices de bondad de ajuste del modelo resultaron satisfactorios para la mitad de la muestra $(n=381): \div 2 / g \mid=2.98, N N F I=.98, C F I=$ .98 y RMSEA $=.072$. Se obtiene además estos índices para hombres y mujeres, nivel y sistema educativo ( $\mathrm{Ta}$ bla 4).

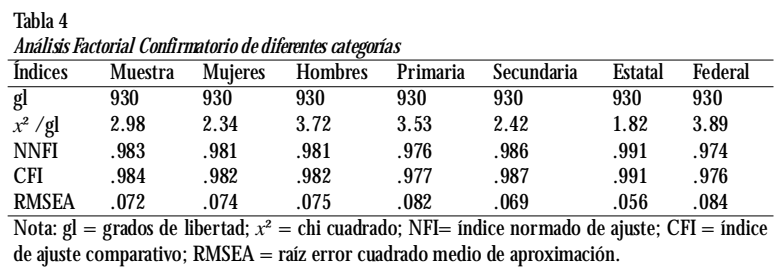

\section{Discusión}

El propósito del presente estudio consistió en analizar la Educación Física de calidad desde la perspectiva de la práctica docente en la República M exicana. En este sentido, se puede señalar que el llevar a cabo el análisis de la información permite contar con un instrumento que se podrá utilizar para evaluar la educación física considerando: las políticas para el mejoramiento de la calidad educativa, la enseñanza de la educación física, desarrollo de habilidades, propósitos y contenidos, los planes para la factibilidad y accesibilidad para todos los estudiantes, y las normas sociales y práctica cultural.

La estructura interna del instrumento y la prueba de esfericidad de Bartlett resultó satisfactoria y estadísticamente significativa, lo que lleva a asumir que la aplicación del análisis es pertinente. Para comprobar la estructura factorial se llevó a cabo el análisis factorial confirmatorio; que de acuerdo con las recomendaciones los índices de bondad de ajuste del modelo del estudio resultaron, de igual forma, satisfactorios. 0 tros estudiosmuestran deigual formaadecuadosíndices, como por ejemplo los obtenidos por Ho et al. (2019) sobre la educación física de calidad en docentes de Asia $(\div 2 / \mathrm{gl}$ $=3.45, \mathrm{TLI}=.92, \mathrm{CFI}=.93, \mathrm{RMSEA}=.078) ;$ Pala cios-Picos et al. (2018) sobre la percepción de competencias docentes de educación física de España $(\div 2 / \mathrm{gl}$ $=3.45, \mathrm{NNFI}=.97, \mathrm{CFI}=.98, \mathrm{RMSEA}=.068)$; Richards et al. (2016) sobre la percepción de la materia deEducación Física(PMQ -PE) en Estados U nidos $(\div 2$ / $\mathrm{gl}=2.50, \mathrm{NNFI}=.97, \mathrm{CFI}=.98, \mathrm{RMSEA}=.07$ ).

Se pueden observar otros estudios que han probado las propiedades psicométricas de instrumentos en educación física, un ejemplo de ello es la adaptación de la 
escala de las necesidades psicológicas básicas en el ejercicio a las clases de educación física en el contexto espa ñol (M oreno, González-Cutre, Chillón \& Parra, 2008), con valores de ajuste aceptables $(\div 2 / \mathrm{gl}=3.29, \mathrm{TLI}=$ $.92, \mathrm{CFI}=.94, \mathrm{RMSEA}=.07)$. Respecto a la versión informatizada del Physical Self Description $Q$ uestionnaire en adolescentes mexicanos ( 0 rnelas, Conchas, Citlalli, Blanco \& Rangel, 2020), evidenciando una consistencia interna adecuada (RMSEA $=.04$; $\mathrm{TLI}=.90 ; \mathrm{CFI}=.97)$.

Los resultados obtenidos en el presente estudio muestran índices satisfactorios, la consistencia interna de cada uno de los factores resultantes fue excelente; así, se demuestra que existe una gran homogeneidad entre los ítems de cada factor (N unnally, 2008). 0 tros autores obtienen datos similares de un instrumento de diagnóstico y valoración del componente evaluativo para docentese instituciones educativas (González, Chaverra, Bustamante \& Toro, 2020).

Este tipo de estudios contribuyen en el cambio social de los últimos años, que provoca mayores demandas en el profesorado del siglo XXI. Los sistemas educativos deben tratar de garantizar que las personas cuenten con las competencias que se requieren en el merca do laboral (OCDE, 2016). Ante esta afirmación, se debe lograr competencias profesionales de las docentes rela cionadas fundamentalmente con el contenido, su forma de enseñarlo, la utilización de tecnologías, el tipo de aprendizaje que se obtiene, la organización del al umnado, las emociones implicadas, entre otras (Levi-0 rta \& Ramos-M éndez, 2013; Sanz, 2010; Zabala \& Arnau, 2014).

Hay que pensar que los docentes requieren de competencias profesionales que les permita enfrentarse al nuevo contexto educativo para que puedan llevar a cabo una educación de calidad incluso en casa ante situaciones de emergencia (Burgueño et al., 2021), surgiendo la necesidad de investigar cuáles son las características que constituyen un profesor eficaz y capaz de hacer frente a las demandas de la sociedad actual (Cañadas, SantosPastor \& Castekon, 2019; Villafuerte, Pérez \& Delga do, 2019). El instrumento utilizado en esta investiga ción contempla estos elementos necesarios e indispensables para construir una educación física de calidad, con especial atención en los países en desarrollo como es el caso de México.

\section{Conclusiones}

La escala de Educación Física de Calidad desde la perspectiva de la práctica docente en México es una herramienta de fácil aplicación y dispone de las suficientes garantías psicométricas para ser utilizada por investigadores, autoridades educativas y profesionales de la educación física en la promoción de: Políticas para el mejoramiento de la calidad educativa, Enseñanza de la educación física, Desarrollo de habilidades, Propósitos y contenidos, Planes para la factibilidad y accesibilidad para todos los estudiantes, Normas sociales y práctica cultural.

En cuanto a las limitaciones de este estudio, se considera que la forma de aplicación puede considerarse una limitación; en virtud de que al ser aplicado de ma nera online pudiera traer confusión en al guna pregunta y no se pudiera dar solución en el momento de ser respondido; sin embargo, también cubre a la vez una demanda específica para poder eval uar la Educación Física de Calidad.

\section{Agradecimientos}

El trabajo contó con un financiamiento del Progra ma de A poyo a la Investigación Científica y Tecnológica (PAICYT) de la Universidad Autónoa de Nuevo León.

\section{Referencias}

BatistaFoguet, J. M. ., Coenders, G. \& Alonso, J. (2004). A nálisis factorial confirmatorio. Su utilidad en lavalidación de cuestionarios relacionados con la salud. M edicina dínica, 122 (1), 2127.

Bentler, P. M. (2005). EQS 6 Manual de programa de eccuaciones estructurales. Software multivariante, Encino.

Burgueño, R., Bonet-M edina, A., Cerván-Cantón, Á, Espejo, R., Fernández-Berguillo, F., Gordo-Ruiz, F., Linares-Martínez, H., M ontenegro-Lozano, S., O rdoñez-Tejero, N.,Vergara-Luque, J. \& Gil-Espinosa, F. (2020). Educación Físicaen CasadeCalidad. Propuesta de aplicación curricular en Educación Secundaria O bligatoria. Retos, 39, 787-793. https:/ / doi. org/ 10.47197/ retos. v0i39. 78792

Byrne, B. (2000). Structural equation modeling with AMOS: Basic concepts, applications, and programming. Mahwah, NJ: LawrenceErlbaum Associates.

Calabuig, F., Q uintanilla, I. \& Mundana, J. (2007). La calidad Percibida de los servicios deportivos: diferencias según insta lación, género, edad y tipo de usuario en servicios náuticos, Revista Internacional de Ciencias del Deporte. 10(IV), 25-43.

Cañadas, L., Santos-Pastor, M. L. \& Castejón, F.J. (2018). Competencias docentes en la formación inicial del profesorado de educación física. Retos, 35, 284-288. https:/ / doi.org/ 10.47197/ retos. v0i35.64812

Coutín, A., Gástelum, G. \& Guedea, J. C. (2018). Problemas ac- 
tuales deloscurrículosen laeducación físicaen Iberoamérica. Una revisión sistemática. Revista Ciencias de la Actividad Física UCM, 19(2), 1-9. http:/ / doi.org/ 10.29035/ rcaf.19.2.1

Cronbach, L. J. (1951). Coefficient alpha and theinternal structure of tests. Psychomerika,(16), 1-16.

Galaviz, K. I., Argumedo, G., M edina, C., Gaytán-González, A., González-Casanova, I.,Villalobos, M. F., ... Pelayo, R.A. (2018). ¡Es hora de hacer latareal Por una educación física de calidad en M éxico. Boleta de Calificaciones M exicana sobrelaActividad FísicadeNiñosy Jóvenes. Guadalajara.

Gil Espinosa, F.J. \&Aznar Cebamanos, M. (2016). Educaciónfísica de calidad en el sistema educativo español. Revista Española de Educación Física y Deportes, 414, 69-82.

González, E., Chaverra, B., Bustamante, S. \&Toro, C. (2020). Dise ño y validación de un cuestionario sobre las concepciones y percepción de los estudiantes sobre la evaluación en Educa ción Física. Retos, 40, 317-325. https:/ / doi.org/ 10.47197/ retos. v1i40.80914

Guthold, R., Stevens, G. A., Riley, L. M. \& Bull, F. C. (2018). Worldwide trends in insufficient physical activity from 2001 to 2016: a pooled analysis of 358 population-based surveys with 1.9 million participants Lancet $G$ lob H ealth, 6(10), e1077e1086. doi.org/ 10.1016/ S2214-109X (18)30357-7

Hambleton, R. K. \& Kanjee, A. (1995). Increasing the validity of cross-cultural assessments: Use of improved methods for test adaptations. European Journal of Psychological Assessment, 11(3), 147-157.

Ho,W., Ahmed, D., Carvalho, P. G., Antala, B., Imre, M., Valeiro, M. G., ... Wong, B. (2019). Development of an instrumentto assess perception of Q uality Physical Education among the European professionals. South African Journal for Research in Sport, Physical Education and Recreation, 41(1), 31-49.

Ho,W.,Ahmed, D., Khoo, S., Tan, C. H., Dehkordi, M. R., Gallardo, M.,... Shu, C. (2019).Towardsdevelopingand validating Q uality Physical Education in schools. The Asian physical education professionals' voice. PLOS ONE 14(8), e0218158. https:/ / doi.org/ 10.1371/ journal. pone. 0218158

Ho,W., Ahmed, D., López-deD'Amico, R., Ramos, A., Ferreira, E., RochaFerreira, E. ... Wong, B. (2018) Measuring the perception of quality physical eduction in Latin American professionals RevistaBrasileiradeCiências do Esporte40(4), 361-369.

Ho,W., Ahmed, D., Wong, B., Huang, F., López-deD'Amico, R., Dinold, M. \& Branisla, A. (2016). Q uality physical education and global concern - ways ahead and future development. Revista Electrónica Activi-dad Física y Cienciass 8(1), 60-70.

Hu, L. \& Bentler, P. M. (1995). Evaluating mode fit. In R. H. Hoyle (Ed.), Structural equation modeling Concepts, issues and applications California, EU: Sage Publications. (37-55).

Jauregui, A. (2018). Hacia una estrategia nacional para la pretación de Educación Físca de calidad en el nivel básco del Sistema Educativo M exicano. Cuernavaca, Instituto Nacional deSalud Pública.

Jöreskog, K. G. \& Sörbom, D. (2006). LISREL 8.80. Scientific Software International. Inc, Copyright.

Kline, R. B. (2005). Principles and practice of structural equationmodeling. (2a Ed.). New York, NY: The Guilford Press.

Levi-O rta, G. \& Ramos-M endez, E. (2013). Componentes delas competencias en los nuevos grados de algunas universidades españolas. Revisa deEducación, 362, 623-658. DO I: 10.4438/ 1988-592X-RE-2013-362-244

López,V. M., Pérez, D., M anrique, J. C. \& M onjas, R. (2016). Los retos de la Educación Física en el Siglo XXI. Retos, 29, 182187.

Moreno, J.A., González-Cutre, C., Chillón, M. \& Parra, N. (2008). Adaptación ala educación físicade laescalade las necesidades psicológicas básicas en el ejercicio. Revista Mexicana de Psicología, 25(2), 295-303.

Nunnally, J. C. (2008). Psychometric theory. Digital New York: McGraw Hill.

O ECD (2016). Education at a Glance 2016: OECD Indicators, OECD Publishing. http:/ / dx. doi.org/ 10.187/ eag-2016-en

O rnelas, M., Conchas, M., Cittalli, A., Blanco, L. H. \& Rangel, S. (2020). Propiedades psicométricas de una versión informatizada del Physical Self D escription Q uestionnaire en adolescentes mexicanos. Retos, 37, 22-26.

0 viedo, H. C. \& Campo-Arias, A. (2005). A pproach to the use of Cronbach's alpha coefficient. Revista Colombiana de Psiquiatría, 34(4), 572-580.

Palacios, A., López-Pastor, V. \& Fraile, A. (2019) Perception Q uestionnaire of Teaching Competences in Physical Education. Revista Internacional de Medicina y Ciencias de la Actividad Físca y e Deporte,19(75), 445-461. DOI: 10.15366/ rimcafd2019.75.005

Reguant-Álvarez, M. \& Torrado-Fonseca, M. (2016). El método Delphi. REIRE, Revista d'Innovació i Recerca en Educació, 9(1), 87-102. DOI: 10.1344/ reire2016.9.1916

Richards, K. A., Gaudreault, K. L. \& Woods, A. M. (2016). Understanding physical educators perceptions of mattering:Validation of thePerceived M atteringQ uestionnaire - Physical Education. European Physical Education Review, 23(1), 1-18. DOI: $10.1177 / 1356336 \times 16637320$

Sanz, C. (2010). La orientación profesional en los sistemas de formación profesional. Revista Española de Orientación y Psicopedagogía, 21(3), 643-652.

Soto, J. I., Collado, D., Torres, B. \& Padial, R. (2021). Elaboración y validación de un cuestionario (CFVALMA), sobre laforma ción en valores del alumnado de magisterio. Retos, 41, 68-77. https:/ / doi.org/ 10.47197/ retos. v0i41.81984

UNESCO (2015). Educación Físca de Calidad. Guía para los responsables políticos. Recuperado de http:/ / unesdoc. unesco.org/ images/ 0023/ 002313/ 231340s. pdf.

Villafuerte, J., Pérez, L. \& Delgado,V. (2019). Retos de laEduca ción Física, Deportes y Recreación en Ecuador: las compe tencias docentes. Retos, 36 (36), 327-335. https:/ / doi.org/ 10.47197/ retos. V36i36.67062

ZabalaA. \& Arnau L. (2014). M étodos para la enseñanza de las competencias. Barcelona, GRAÓ. 